\title{
Fast Evaluation of Scattering Oscillatory Integrals Over Fan-Shaped Regions
}

\author{
Jianbing Li* Tao Wang and Xuesong Wang
}

State Key Laboratory of Complex Electromagnetic Environmental Effects on Electronics \& Information System, School of Electronic Science and Engineering, National University of Defense Technology, Changsha, Hunan, China

Received: 30 Aug. 2013, Revised: 27 Nov. 2013, Accepted: 28 Nov. 2013

Published online: 1 Sep. 2014

\begin{abstract}
Oscillatory integrals over fan-shaped regions widely exist in scattering analysis, and this paper proposes a new rapid calculation routine for them. In the routine, the concerned bivariate scattering oscillatory integral is reduced to the calculation of two univariate oscillatory integrals. One is calculated with the improved Levin quadrature method, and another is transformed to a non-oscillatory integral that can be stably and accurately calculated with the Gauss-Chebyshev integration rule. Numerical examples show that the newly proposed method has the merits of being very accurate and efficient.
\end{abstract}

Keywords: Oscillatory integral, Fan-shaped integration region, Chebyshev spectral method, Gauss-Chebyshev integration rule

\section{Introduction}

Due to the high oscillations of the incident wave and the Green function $G\left(\mathbf{r}, \mathbf{r}^{\prime}\right)=\mathrm{e}^{\mathrm{i} k\left|\mathbf{r}-\mathbf{r}^{\prime}\right|} / 4 \pi\left|\mathbf{r}-\mathbf{r}^{\prime}\right|$, we often need to compute integrals of oscillatory type in electromagnetic scattering simulations $[1,2,3,4,5]$ :

$$
I=\iint_{S} f\left(k, \mathbf{r}^{\prime}\right) \mathrm{e}^{\mathrm{i} k g\left(k,\left|\mathbf{r}-\mathbf{r}^{\prime}\right|\right)} \mathrm{d}^{2} \mathbf{r}^{\prime}
$$

where $S$ denotes the surface of the object, $k$ is wavenumber of the incident wave, $\mathbf{r}$ and $\mathbf{r}^{\prime}$ are vectors of the field point and source point, $f\left(k, \mathbf{r}^{\prime}\right)$ is called the amplitude function, and $g\left(k,\left|\mathbf{r}-\mathbf{r}^{\prime}\right|\right.$ ) (generated from the Green function) is called the phase function. For example, when estimating the RCS of a perfect electrical conductor using the physical optics method, the integral to be calculated can be abstracted as[1]

$$
I_{\mathrm{po}}=\iint_{S_{\mathrm{lit}}} \hat{\mathbf{k}} \cdot \hat{\mathbf{n}}\left(\mathbf{r}^{\prime}\right) \mathrm{e}^{\mathrm{i} 2 \mathbf{k} \cdot \mathbf{r}^{\prime}} \mathrm{d} s
$$

where $S_{\text {lit }}$ is the lit region, $\mathbf{k}$ is the wavenumber vector, $\hat{\mathbf{k}}$ is the unit vector of $\mathbf{k}, \mathbf{r}^{\prime}$ is the vector of a point on the surface, and $\hat{\mathbf{n}}\left(\mathbf{r}^{\prime}\right)$ is the outward normal vector at $\mathbf{r}^{\prime}$. It is noted that for different scattering analysis method we shall have different expressions of the amplitude function and phase function.
For scattering problems at high frequencies (large $k$ ), this kind of integral is generally very difficult to calculate with conventional quadrature methods such as the Simpson quadrature and Gauss quadrature[6], so efforts have been made in the past decades to find rapid calculation routines for them[7,8]. In $[9,10,11,12] \mathrm{Li}$ et al. proposed a delaminating quadrature method to calculate multi-dimensional highly oscillatory integrals based on rectangular integration regions. But in practise we often have to handle oscillatory integral over fan-shaped integration regions. This paper concerns the scattering oscillatory integrals over fan-shaped regions, and proposes a new rapid calculate routine for them.

\section{New routine to calculate scattering oscillatory integrals over fan-shaped regions}

\subsection{Scattering Oscillatory Integrals Over Fan-shaped Regions}

The scattering oscillatory integral over a fan-shaped integration region $\left[\theta^{-}, \theta^{+}\right] \times[0, R]$ can be written as

$$
I=\int_{0}^{R} \int_{\theta^{-}}^{\theta^{+}} f(\theta, \rho) \mathrm{e}^{\mathrm{i} k g(\theta, \rho)} \mathrm{d} \rho \mathrm{d} \theta .
$$

\footnotetext{
*Corresponding author e-mail: jianbingli@nudt.edu.cn, ljbnudt@gmail.com
} 
For simplicity the variable $k$ in the amplitude function and phase function has been omitted since it remains constant for a fixed incident wave. Of course, a general fan-shaped region $\left[\theta^{-}, \theta^{+}\right] \times[0, R(\theta)]$ can also be transformed to the above integration region if the coordinate transformation $\rho=R(\theta) \rho^{\prime}$ is used, so this paper pays the main attention on the calculation of oscillatory integral as shown in (3). In addition, the phase function in a scattering problem is generated from the Green function $G\left(\mathbf{r}, \mathbf{r}^{\prime}\right)=\mathrm{e}^{\mathrm{i} k\left|\mathbf{r}-\mathbf{r}^{\prime}\right|} / 4 \pi\left|\mathbf{r}-\mathbf{r}^{\prime}\right|$. In this sense, $\rho=0$ corresponds to the case that the source point $\mathbf{r}^{\prime}$ coincides with the local coordinate origin, and then the phase function $g(\theta, 0)$ will remain constant for different $\theta$.

\subsection{The Impact of Critical Points}

It is also noted that the critical points (stationary point and resonant point) have to be carefully handled when calculating oscillatory integrals with the delaminating quadrature[9]. A resonant point (also called the boundary critical point) is a point where the gradient of phase function $\left(\nabla g(\theta, \rho)=\left(\frac{1}{\rho} \frac{\partial g(\theta, \rho)}{\partial \theta}, \frac{\partial g(\theta, \rho)}{\partial \rho}\right)\right.$ is orthogonal to the boundary, and a stationary point (also called the inner critical point) is a point where the gradient of phase function vanishes. As analyzed in [9], the delaminating quadrature method works well for oscillatory integrals with critical points on the vertices of the integration regions since the Chebyshev spectral method samples densely around the vertices. In this sense, if critical point is involved, the division of integration region is proposed to make the critical points locate on the vertices. Fig. 1 presents the schemes of region division with respect to the locations of the critical points.

1.the case free of critical point (see Fig. 1(a)): no region division is required for this case.

2.the case with a resonant point on the arc (see Fig. 1(b)): in this case, the original fan-shaped region is divided into two fan-shaped regions (I and II).

3.the case with a resonant point on the radius (see Fig. 1(c)): in this case the region is divided into a fan-shaped region (I) and a wedge (II).

4.the case with a stationary point (see Fig. 1(d)): in this case the original region is divided into two fan-shaped regions (I and II) and two wedges (III and IV).

5.the case with multiple critical points: combination of the above division scheme is proposed in this case.

After the division, the critical points are all located on the vertices of the subregions, and each subregion is free of inner and boundary critical point(s). Since the delaminating quadrature method works well for oscillatory integral over wedge regions, the following section pays the main attention on the fan-shaped regions (region I for case 1, regions I and II for case 2, region I for case 3, regions I and II for case 4).
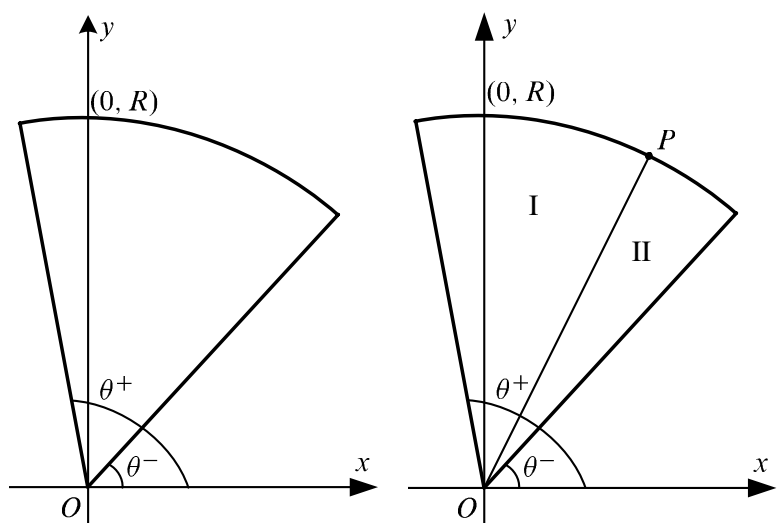

(a) The case free of critical (b) The case with a resonant point point on the arc
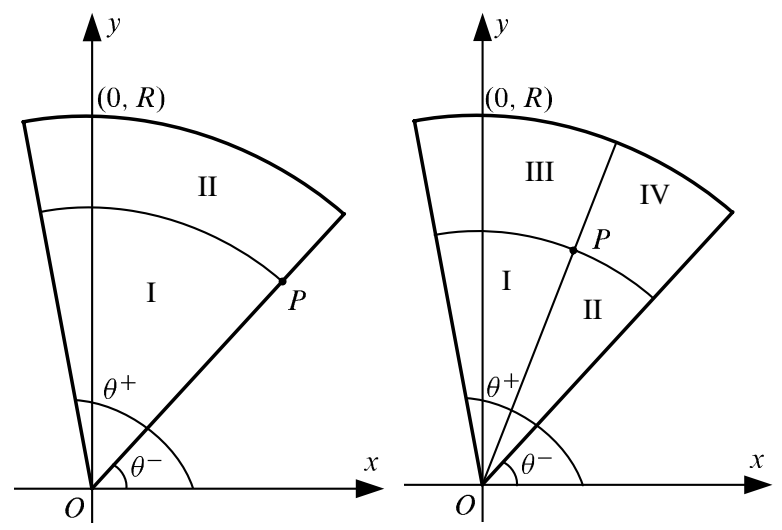

(c) The case with a resonant(d) The case with a stationary point on the radius phase point

Fig. 1: Sub-division of the integration region.

\subsection{New calculation routine}

Without lose of generality, we study integral (3). We first study the integration about variable $\rho$. According to the Levin method, it can be reduced to finding a function $p(\theta, \rho)$ to satisfy the following differential equation[9]:

$$
\frac{\partial p(\theta, \rho) \mathrm{e}^{\mathrm{i} k g(\theta, \rho)}}{\partial \rho}=f(\theta, \rho) \mathrm{e}^{\mathrm{i} g(\theta, \rho)},
$$

which is equivalent to

$$
\left.p_{\rho}^{\prime}(\theta, \rho)+\mathrm{i} k g_{\rho}^{\prime}(\theta, \rho)\right) p(\theta, \rho)=f(\theta, \rho) .
$$

Substituting (5) or (4) into (3) gives

$$
\begin{aligned}
I & =\int_{\theta^{-}}^{\theta^{+}} p(\theta, R) \mathrm{e}^{\mathrm{i} k g(R, \theta)} \mathrm{d} \theta-\int_{\theta^{-}}^{\theta^{+}} p(\theta, 0) \mathrm{e}^{\mathrm{i} k g(\theta, 0)} \mathrm{d} \theta \\
& \triangleq I_{1}-I_{2} .
\end{aligned}
$$

Therefore the original bivariate oscillatory integral is transformed into two univariate oscillatory integrals. 
In (6) the first integral $I_{1}$ can be calculated with the improved Levin method proposed in [12,9], but the second one $\left(I_{2}\right)$ is a little special. As mentioned in section 2.2, the phase function $g(\theta, 0)$ in scattering analysis remains constant for different values of $\theta$. In this sense, the integral $I_{2}$ is in fact not an oscillatory integral, and the factor $\mathrm{e}^{\mathrm{i} k g(\theta, 0)}$ can be directly extracted from the integral:

$$
I_{2}=\mathrm{e}^{\mathrm{i} k g(\theta, 0)} \int_{\theta^{-}}^{\theta^{+}} p(\theta, 0) \mathrm{d} \theta
$$

This integral is non-oscillatory, so it can be efficiently calculated with a conventional quadrature method such as the Gauss integration rule[6], and then the integral $I=I_{1}-I_{2}$ is obtained.

The detailed procedures of this routine are as follows:

1.Solve the differential equaiton (5): At a given sample of $\theta: \theta_{n}=\frac{\theta^{+}-\theta^{-}}{2} \cos \frac{n \pi}{N_{\theta}}+\frac{\theta^{+}+\theta^{-}}{2}, n=0,1, \ldots, N_{\theta}$, we can solve the differential equation (5) using the Chebyshev spectral method to get the unknown function values $p(\theta, \rho)$ at giving radius samples $\left(\rho_{m}=\frac{R}{2}\left(\cos \frac{m \pi}{N_{\rho}}+1\right), m=0,1, \ldots, N_{\rho}\right)[9]:$

$$
\left(\frac{2}{R} \mathbf{D}_{N_{\rho}}+\mathrm{i} k \mathbf{G}_{n}^{\rho}\right) \mathbf{P}_{n}=\mathbf{F}_{n},
$$

where $\mathbf{D}_{N_{\rho}}$ is the Chebyshev differentiation matrix of order $N_{\rho}, \mathbf{F}_{n}=\left[f\left(\theta_{n}, \rho_{0}\right), f\left(\theta_{n}, \rho_{1}\right), \ldots, f\left(\theta_{n}, \rho_{N_{\rho}}\right)\right]^{\mathrm{T}}$ is a numerical vector related to the amplitude function, $\mathbf{G}_{n}^{\rho}$ is a diagonal matrix: $\mathbf{G}_{n}^{\rho}=\operatorname{diag}\left(g_{\rho}^{\prime}\left(\theta_{n}, \rho_{0}\right), g_{\rho}^{\prime}\left(\theta_{n}, \rho_{1}\right), \ldots, g_{\rho}^{\prime}\left(\theta_{n}, \rho_{N_{\rho}}\right)\right)$, and $\mathbf{P}_{n}=\left[p\left(\theta_{n}, \rho_{0}\right), p\left(\theta_{n}, \rho_{1}\right), \ldots, p\left(\theta_{n}, \rho_{N_{\rho}}\right)\right]^{\mathrm{T}}$ is a numerical vector to be computed. For different $\theta_{n}$ we shall get different $\mathbf{P}_{n}$ from (8), and the combination of them give a numerical matrix $\mathbf{P}=\left\{\mathbf{P}_{0}, \mathbf{P}_{1}, \ldots, \mathbf{P}_{N_{\theta}}\right\}$.

2.Calculate the integral $I_{1}$ : The calculation of $I_{1}$ can be reduced to solving the following system of linear equations[9]:

$$
\left(\frac{2}{\theta^{+}-\theta^{-}} \mathbf{D}_{N_{\theta}}+\mathrm{i} k \mathbf{G}_{R}^{\theta}\right) \mathbf{Q}=\mathbf{P}^{+},
$$

where $\mathbf{D}_{N_{\theta}}$ is the Chebyshev differentiation matrix of order $N_{\theta}, \mathbf{Q}=\left[q\left(\theta_{0}, R\right), q\left(\theta_{1}, R\right), \ldots, q\left(\theta_{N_{\theta}}, R\right)\right]^{\mathrm{T}}$ is an unknown numerical vector, $\mathbf{G}_{R}^{\theta}$ is a diagonal matrix:

$\mathbf{G}_{R}^{\theta}=\operatorname{diag}\left(g_{\theta}^{\prime}\left(\theta_{0}, R\right), g_{\theta}^{\prime}\left(\theta_{1}, R\right), \ldots, g_{\theta}^{\prime}\left(\theta_{N_{\theta}}, R\right)\right)$, and $\mathbf{P}^{+}$is the transpose of the first row of matrix $\mathbf{P}$. When the unknown numerical vector $\mathbf{Q}$ is calculated from (9), the integral $I_{1}$ in (6) is obtained as

$$
I_{1}=\mathbf{Q}_{0} \mathrm{e}^{\mathrm{i} k g\left(\theta^{+}, R\right)}-\mathbf{Q}_{N_{\theta}-1} \mathrm{e}^{\mathrm{i} k g\left(\theta^{-}, R\right)}
$$

3. Since we have obtained the function values of $p(\theta, 0)$ at Chebyshev-Lobatto $\left(p\left(\theta_{n}, 0\right), n=0,1, \ldots, N_{\theta}\right)$, we shall calculate the integral $I_{2}$ with the Gauss-Chebyshev integration rule of the second kind[13]. However, the Gauss-Chebyshev quadrature omits the function values at the endpoints, so its accuracy is generally not satisfactory for a integral with non-zero integrand at the endpoints. In order to improve the accuracy of integration, we transform the above integral as follows:

$$
\begin{aligned}
I_{2} & =\mathrm{e}^{\mathrm{i} k g(\theta, 0)}\left\{\int_{\theta^{-}}^{\theta^{+}}[p(\theta, 0)-h(\theta)] \mathrm{d} \theta+\int_{\theta^{-}}^{\theta^{+}} h(\theta) \mathrm{d} \theta\right\} \\
& \triangleq \mathrm{e}^{\mathrm{i} k g(\theta, 0)}\{\underbrace{\int_{\theta^{-}}^{\theta^{+}} \kappa(\theta) \mathrm{d} \theta}_{I_{2}^{\prime}}+\underbrace{\int_{\theta^{-}}^{\theta^{+}} h(\theta) \mathrm{d} \theta}_{I_{2}^{\prime \prime}}\} .
\end{aligned}
$$

where $h(\theta)=p\left(\theta^{-}, 0\right)+\frac{p\left(\theta^{+}, 0\right)-p\left(\theta^{-}, 0\right)}{\theta^{+}-\theta^{-}}\left(\theta-\theta^{-}\right)$is a linear function connecting the two endpoints $\left(\left(\theta^{-}, p\left(\theta^{-}, 0\right)\right)\right.$ and $\left.\left(\theta^{+}, p\left(\theta^{+}, 0\right)\right)\right)$. In (11) the integrand $\kappa(\theta)=p(\theta, 0)-h(\theta)$ vanishes at each endpoint, so the Gauss-Chebyshev integration will be very accurate for it.

(a)Calculation of integral $I_{2}^{\prime}$ : We first employ the coordinate transformation $\theta=\frac{\theta^{+}-\theta^{-}}{2} t$ to transform the integration interval to a standard one:

$$
I_{2}^{\prime}=\frac{\theta^{+}-\theta^{-}}{2} \int_{-1}^{1} \kappa\left(\frac{\theta^{+}-\theta^{-}}{2} t\right) \mathrm{d} t,
$$

and then applying the Gauss-Chebyshev integration rule to the integral gives

$$
\begin{aligned}
I_{2}^{\prime} & =\frac{\theta^{+}-\theta^{-}}{2} \int_{-1}^{1} \sqrt{1-t^{2}}\left\{\frac{\kappa\left(\frac{\theta^{+}-\theta^{-}}{2} t\right)}{\sqrt{1-t^{2}}}\right\} \mathrm{d} t \\
& \approx \frac{\theta^{+}-\theta^{-}}{2} \sum_{n=1}^{N_{\theta}-1} w_{n} \frac{\kappa\left(\frac{\theta^{+}-\theta^{-}}{2} t_{n}\right)}{\sqrt{1-t_{n}^{2}}} .
\end{aligned}
$$

Since the weights and nodes of Gauss-Chebyshev quadrature of the second kind are known: $w_{n}=\frac{\pi}{N_{\theta}} \sin ^{2} \frac{n \pi}{N_{\theta}}$ and $t_{n}=\cos \frac{n \pi}{N_{\theta}}$, we have

$$
\frac{w_{n}}{\sqrt{1-t_{n}^{2}}}=\frac{\pi}{N_{\theta}} \sin \frac{n \pi}{N_{\theta}}, n=1,2, \cdots, N_{\theta}-1 .
$$

The function values $p\left(\frac{\theta^{+}-\theta^{-}}{2} t_{n}, 0\right)$ have been computed in (8), so the function value samples $\kappa\left(\frac{\theta^{+}-\theta^{-}}{2} t_{n}\right)=p\left(\frac{\theta^{+}-\theta^{-}}{2} t_{n}, 0\right)-h\left(\frac{\theta^{+}-\theta^{-}}{2} t_{n}\right)$ are easy to calculate, and then the integral $I_{2}^{\prime \prime}$ is obtained.

(b)Calculation of integral $I_{2}^{\prime \prime}$ : The integral $I_{2}^{\prime \prime}$ can be obtained in a closed form:

$$
I_{2}^{\prime \prime}=\frac{\left[p\left(\theta^{+}, 0\right)+p\left(\theta^{-}, 0\right)\right]\left(\theta^{+}-\theta^{-}\right)}{2} .
$$


Then the integral $I_{2}$ is obtained as: $I_{2}=\mathrm{e}^{\mathrm{i} k g(\theta, 0)}\left(I_{2}^{\prime}+I_{2}^{\prime \prime}\right)$.

4.Finally, the target integral result is obtained as $I=I_{1}-$ $I_{2}$.

\section{Numerical Examples}

This section presents two numerical examples to test the performance of the newly proposed method: an oscillatory integral free of critical point and another one with critical points.

\subsection{Example 1}

In this example we take the physical optics (PO) approximation (2) to test the performance of the newly proposed method. The RCS estimated by the PO approximation follows

$$
\sigma_{\mathrm{PO}}=\frac{k^{2}|I|^{2}}{\pi}
$$

where the PO integral $I=\iint_{S_{\text {lit }}} \hat{\mathbf{k}} \cdot \hat{\mathbf{n}} \mathrm{e}^{\mathrm{i} 2 \mathbf{k} \cdot \mathbf{r}^{\prime}} \mathrm{d} s$ is an oscillatory integral.

We consider a fan-shaped integration region as shown in Fig. 2, and the target PO integral can then be rewritten as

$$
I=\int_{\theta^{-}}^{\theta^{+}} \int_{0}^{R} \rho \cos \beta \mathrm{e}^{\mathrm{i} 2 k \rho \sin \beta \cos (\theta-\phi)} \mathrm{d} \rho \mathrm{d} \theta .
$$

Let $\phi=\pi / 6, \beta=\pi / 6, R=3, \theta^{-}=-\pi / 4, \theta^{+}=\pi / 6$, and 50 incident frequency samples range from $5 \mathrm{GHz}$ to $10 \mathrm{GHz}$ evenly. Under this situation, the integral is free of critical point, and no region division is required.

During the calculation, the numbers of nodes used in the calculation are $N_{\rho}=N_{\theta}=25$. The performance of the proposed method is presented in Fig. 3, which is a double y-axis plot with the left side axis denoting the RCS and the right side axis denoting the relative error of this approach.

From the results it is observed that the RCS obtained by the new method coincides well with that of Gauss quadrature, and the relative errors of the proposed method are very small $\left(\mathscr{O}\left(10^{-5}\right)\right.$ or even smaller), so the new method is very accurate for this example. At the same time, the electrical size of the integration region is $\mathscr{O}\left(10^{2}\right)$ at frequency $9 \mathrm{GHz}$, so a conventional quadrature has to use $\mathscr{O}\left(10^{3}\right)$ or more grids in each dimension to calculate the mentioned PO integral accurately. But for the present method, we can get very accurate result with only 25 nodes in each dimensional, so the new method is also very efficient.

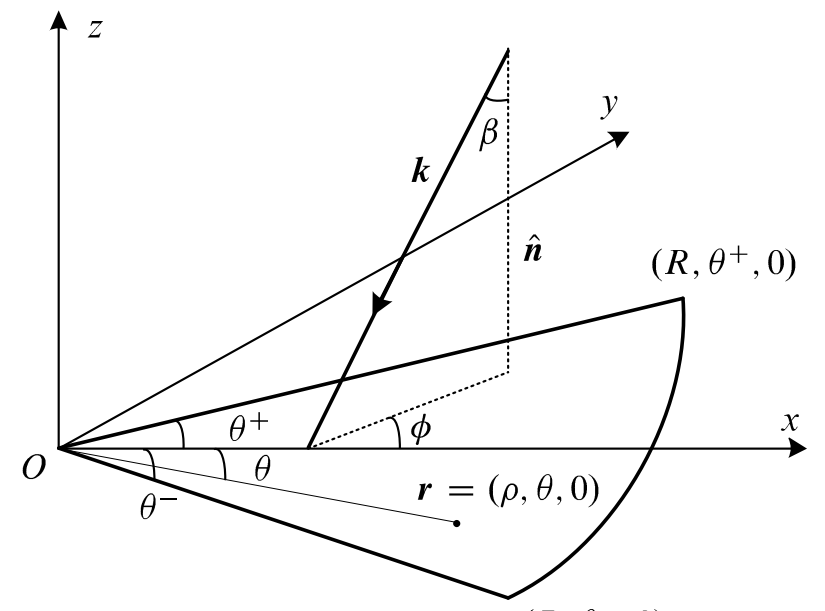

$\left(R, \theta^{-}, 0\right)$

Fig. 2: Geometry relation for example 1.

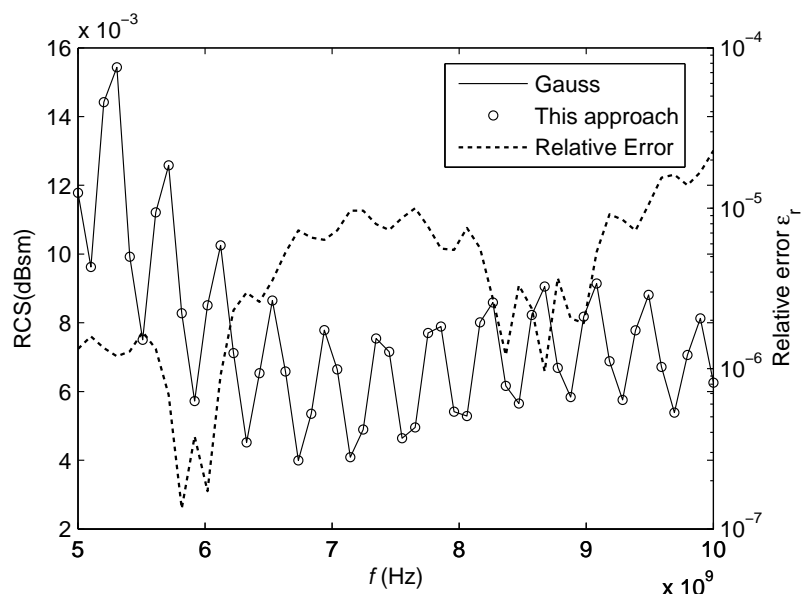

Fig. 3: Performances of the new method for scattering oscillatory integral free of critical point.

\subsection{Example 2}

In this example, we study the performance of this method for scattering oscillatory integrals with critical points. Here the PO integral is also taken as the target oscillatory integral, but the amplitude function is set to a more complex one:

$$
\begin{aligned}
f(\theta, \rho)= & (\sin \theta+\cos \theta) . \\
& \cdot\left[1+\mathrm{i} k(\rho+1)\left(\frac{\sin \theta}{2}+\frac{\sqrt{3} \cos \theta}{2}-2 \sqrt{3} \rho\right)\right] .
\end{aligned}
$$

Assume the concerned surface is governed by a function $z=s(x, y)=9-x^{2}-y^{2}$ and which projects to a fan-shaped integration region (see Fig. 4). Let $\phi=\pi / 6$, $\beta=\pi / 6, R=2, \theta_{-}=-\pi / 3, \theta^{+}=2 \pi / 5$, and 50 
incident frequency samples range from $3 \mathrm{GHz}$ to $5 \mathrm{GHz}$ evenly. Under this situation the phase function can be rewritten as $g(\theta, \rho)=2(\rho \cos \theta \sin \beta \cos \phi+$ $\rho \sin \theta \sin \beta \sin \phi+\cos \beta \cdot s(\rho \cos \theta, \rho \sin \theta))$, and there are three critical points: a stationary phase point at $P_{\mathrm{S}}=\left(\theta_{\mathrm{s}}, \rho_{\mathrm{s}}\right)=\left(\frac{\pi}{6}, \frac{\sqrt{3}}{6}\right)$, a resonant point on the arc $P_{\mathrm{r}}^{1}=\left(\theta_{\mathrm{r}}^{1}, \rho_{\mathrm{r}}^{1}\right)=\left(\frac{\pi}{6}, \rho_{\mathrm{r}}^{1}\right)$, and a resonant point on the radius $P_{\mathrm{r}}^{2}=\left(\theta_{\mathrm{r}}^{2}, \rho_{\mathrm{r}}^{2}\right)=\left(\theta^{+}, \frac{\cos \theta^{+}}{4}+\frac{\sqrt{3} \sin \theta^{+}}{12}\right)$.

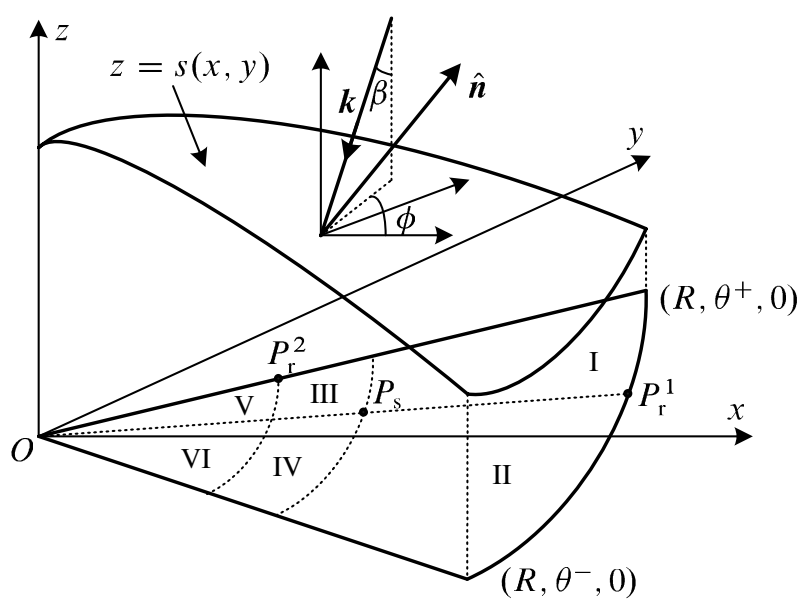

Fig. 4: Geometry relation for example 2.

In order to test the impact of critical points on the new method, two calculation schemes are employed.

-Scheme 1: no region division is used, and the numbers of nodes adopted in the calculation are $N_{\rho}=N_{\theta}=50$.

-Scheme 2: the integration region is divided into six sub-regions as shown in Fig. 4 (I, II, III, IV, V, VI), and the numbers of nodes used are $N_{\rho}=N_{\theta}=25$, which are half of those in scheme 1.

The results are presented in Fig. 5 with the left side axis denoting the magnitude of integral $|I|$ and the right side axis denoting the corresponding relative errors.

From Fig. 5 it is observed that if no region division is adopted (scheme 1), the magnitude of integral (o) does not coincide with the result of Gauss quadrature $(-)$ and the relative error is about $\mathscr{O}(1)$, so the accuracy of this scheme is very poor. However, if the division of integration region is adopted (scheme 2), the method can get very accurate integral results even if only small number of nodes are used (the magnitude of integral $(\bullet)$ well coincides with the result of Gauss quadrature ( - ), and the the relative errors are about $\left.\mathscr{O}\left(10^{-6}\right)\right)$. This phenomenon indicates that, if the division of integration region is employed, the new method can also work well for scattering oscillatory integrals with critical points.

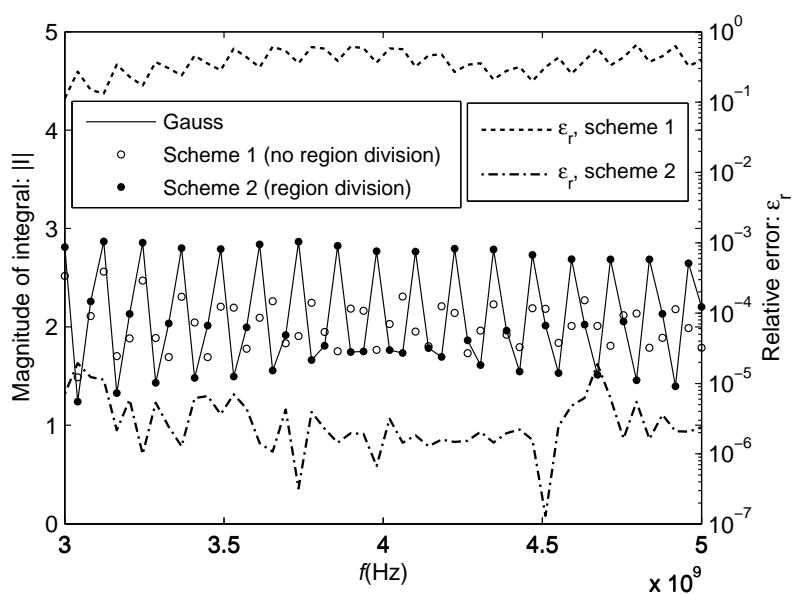

Fig. 5: Performances of the new method for scattering oscillatory integral with critical points.

\section{Acknowledgement}

The work was supported by National Natural Science Foundation of China (No. 61201333 and No. 41375040).

The authors are grateful to the anonymous referee for a careful checking of the details and for helpful comments that improved this paper.

\section{References}

[1] J. Li, X. Wang, and L. Qu, "Calculation of Physical Optics Integrals over NURBS Surface Using a Delaminating Quadrature Method," IEEE Transactions on Antennas and Propagation, 60, 2388-2397 (2012).

[2] J. Zhang, W.M. Yu, X.Y. Zhou, and T.J. Cui, "Efficient Evaluation of the Physical-Optics Integrals for Conducting Surfaces Using the Uniform Stationary Phase Method," IEEE Transactions on Antennas and Propagation, 60, 23982408 (2012).

[3] W.C. Chew, J.M. Jin, C.C. Lu, E. Michielssen, and J.M. Song, "Fast solution methods in electromagnetics," IEEE Transaction on Antenna and Propagation, 45, 533-543 (1997).

[4] J. Li, X. Wang, T. Wang, J. Li, S. Xiao, and Z. Liu, "High range resolution profile of simulated aircraft wake vortices," IEEE Transactions on Aerospace and Electronic Systems, 48, 116-129 (2012).

[5] J. Li, X. Wang, and T. Wang, "Modeling of aircraft wake vortices' dielectric constant distribution," IEEE Transactions on Aerospace and Electronic Systems, 42, 820-831 (2011).

[6] D. Kincaid and W. Cheney, Numerical Analysis: Mathematics of Scientific Computing, Brooks/Cole, Pacific Grove, (2002).

[7] S. Olver, Numerical Approximation of Highly Oscillatory Integrals, phd thesis, University of Cambridge, Cambridge, (2008). 
[8] A. Asheim, Numerical Methods for Highly Oscillatory Problems, Ph.D. thesis, Norwegian University of Science and Technology, Trodheim, (2010).

[9] J. Li, X. Wang, T. Wang, and C. Shen, "Delaminating quadrature method for multi-dimensional highly oscillatory integrals," Applied Mathematics and Computation, 209, 327-338 (2009).

[10] J. Li, X. Wang, T. Wang, and B. Pang, "On a quadrature method for oscillatory integrals," Journal of Mathematical Analysis and Applications, 380, 467-474 (2011).

[11] J. Li, X. Wang, T. Wang, and S. Xiao, "An improved levin quadrature method for highly oscillatory integrals," Applied Numerical and Mathematics, 60, 833-842 (2010).

[12] J. Li, X. Wang, and T. Wang, "A universal solution to onedimensional highly oscillatory integrals," Science in China (series F), 51, 1614-1622 (2008).

[13] P.J. Davis and P. Rabinowitz, Methods of Numerical Integration, Academic Press, New York, (1984).

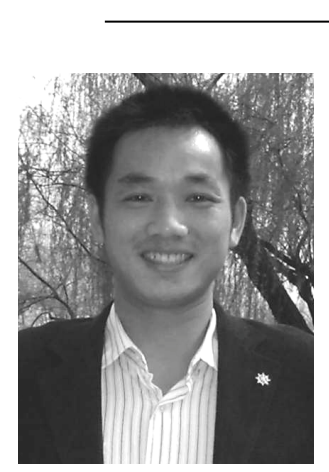

Jianbing Li was born in 1979. He received his B.E. and M.E. degrees from the College of Aerospace and Material Engineering, National University of Defense Technology (NUDT), China in 2002 and 2004, respectively. He earned the Ph.D. degree in December 2010 for his work on the analysis of wake vortices' scattering characteristics from the College of Electronic Science and Engineering, NUDT. His Ph.D. dissertation was awarded as one of the excellent Ph. D. dissertations in Hunan Province in 2011, and which is submitted to compete the national excellent dissertation in China in 2013. He is currently working as an associate professor in the College of Electronic Science and Engineering, NUDT, and his research interests concentrate on radar signal processing and computational electromagnetics.

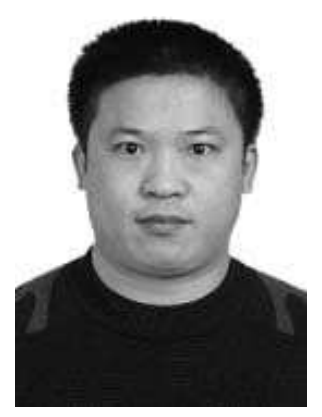

Tao Wang was born in 1976. He earned the bachelors degree in 1999, masters degree in 2001, and Ph.D. in 2006, all in the Electronic and the Information Engineering fields, and all from the National University of Defense Technology, P.R. China. He is now an associate professor in the College of Electrical Science and Engineering at the National University of Defense Technology. His research interests include radar target recognition and polarization information processing.

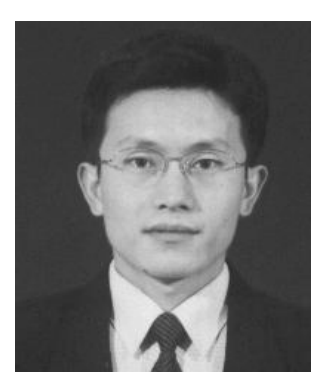

Xuesong Wang was born in 1972. He received his B.E. and Ph.D. degrees from the College of Electronic Science and Engineering, National University of Defense Technology (NUDT), China in 1994 and 1999, respectively. His Ph.D. dissertation was awarded as one of the 100 best Ph.D. dissertations in China in 2001 (two years after his graduation). He is currently working as a professor at NUDT. His research interests concentrate on radar information processing and target recognition. Dr. Wang is also a senior member of the Chinese Institute of Electronics (CIE). 